TITLE:

\title{
The multiple inverse method: a new technique to separate stresses from heterogeneous fault-slip data
}

\author{
AUTHOR(S): \\ Yamaji, Atsushi
}

\section{CITATION:}

Yamaji, Atsushi. The multiple inverse method: a new technique to separate stresses from heterogeneous fault-slip data. Journal of Structural Geology 2000, 22(4): 441-452

\section{ISSUE DATE:}

2000-04-07

URL:

http://hdl.handle.net/2433/139769

\section{RIGHT:}

(c)2000 Elsevier; この論文は出版社版でありません。引用の際には出版 社版をご確認ご利用ください。; This is not the published version.

Please cite only the published version. 


\title{
The multiple inverse method: a new technique to separate stresses from heterogeneous fault-slip data
}

\author{
ATSUSHI YAMAJI \\ Division of Earth and Planetary Sciences, Graduate School of Science, \\ Kyoto University, Sakyo-ku, Kyoto 606-8502, Japan \\ Journal of Structural Geology, 22, 441-452. \\ DOI:10.1016/S0191-8141(99)00163-7.
}

\begin{abstract}
A new inverse technique is presented here to separate stresses from heterogeneous fault-slip data without a priori information on the stresses nor on the classification of faults according to the stresses. Four parameters are determined by the inversion: one for the shape of stress ellipsoid and three for the direction of stress axes. Accordingly, the inversion is equivalent to the projection of fault-slip data to a point in four-dimensional parameter space. The data are divided into $k$-element subsets to which inverse technique is applied, where $k=4$ or 5 is the optimal choice for the stability of solutions and for the reduction of computation. Significant solutions are identified as the clusters in the parameter space. The technique is demonstrated first by simulated fault-slip data. And, as an example, field data obtained from Miocene fore-arc sediments in western Japan were processed by the method.
\end{abstract}

\section{INTRODUCTION}

Advancement in our knowledge about in-situ and paleo stresses has revealed complicated lithospheric stresses in space and time (Engelder, 1993). Deviatoric stresses are calculated by inverse techniques from field observations of the orientation of striations on exposed fault surfaces (Carey and Brunier, 1974; Angelier, 1979; Etchecopar et al., 1981; Gephart and Forsyth, 1984). Such techniques usually assume that all faults slipped in response to the same deviatoric stress. If this is not true, fault-slip data are called heterogeneous. There is a delicate problem for the techniques to process such data. Given a certain sort of field observations allow to classify faults by the stresses that activated the faults, inverse methods can readily identify the stresses (e.g., Angelier and Huchon, 1987). However, such classification is usually difficult, and can introduce a bias into the results.

Several researchers have presented methods to separate stresses from heterogeneous fault-slip data. If we have a priori knowledge that faults compose a conjugate set, Huang's (1988) simple method is appropriate. However, not all deformations result in simple conjugate sets. Moreover, conjugate faults result only in plane strains, but strains are generally three-dimensional (Reches, 1978). Inverse techniques such as Angelier's (1979) can determine triaxial stresses. Armijo et al. (1982) apply inverse calculation recursively to those subset of data that show large misfit from slip directions predicted from formerly determined stresses. The first inversion is applied to the whole fault collection and the data are subdivided into subsets several times. Given heterogeneous data set, however, the subdivision is difficult. Taking this approach Mino and Yamaji (1999) attempted to separate stresses from Quaternary fault data, but second inversion resulted in unstable solutions.

Inverse methods have other difficulties. The assumption of parallelism between striae and maximum shear stress on the fault plane is a matter of debate (Reches, 1978; Marret and Allmendinger, 1990; Pollard et al., 1993). Twiss and Unruh (1998) show that they are parallel if fault block rotations are negligible and if stress and strain are linearly related. Stress inversion is useful only in limited cases in nature. In addition, the interaction among faults causes deviation (Pollard et al., 1993; Cashman and Ellis, 1994; Nieto-Samaniego and Alaniz-Alvarez, 1997).

Great earthquake can leave curved fault striae such as those on the fault plane of the 1995 Hyogo-ken Nanbu (Kobe) earthquake (Yosida et al., 1996; Otsuki et al., 1997). In those cases, coseismic slip direction changes spatially and temporarily with the change of local shear traction across the fault plane (Guatteri and Spudich, 1998), resulting in heterogeneous fault-slip data. Accordingly, inverse methods that can process heterogeneous data adequately are needed.

The aim of this paper is to present a new technique to separate deviatoric stresses from heterogeneous fault-slip data without a priori information on the stresses. Recently, Fry (1999) presents a method to visualize to what extent a possible common stress tensor is constrained by a set of data. He shows that the heterogeneity can be represented by a sort 
of clusters in six-dimensional parameter space. Possible stresses are identified by the present method as clusters in four-dimensional one, and are visualized as clusters on ordinally stereonets. In addition, the reliability of each stress is shown by the density of the cluster. First, the method is tested by an artificial data set, and then applied to field data. A classic inverse method is applied to the same data and the results are compared in the final section.

\section{METHOD}

\subsection{Classic inverse method}

The present method is based on the classic inverse technique by Angelier (1984). Let us use the sign convention that positive and negative stresses correspond to compression and extension, respectively. The inverse method assumes that faults slip in the direction of shear stress acting on the fault plane (Angelier, 1979, 1984). Suppose that we have a number of $N$ fault-slip data, and all the fault slipped under the stress $\sigma$. Let $\mathbf{n}^{\alpha}$ be the unit vector normal to the $\alpha$ th fault $(\alpha=1,2, \ldots, N)$. The vector points into the hanging wall block, so that the stress vector acting on the block is $\mathbf{t}^{\alpha}=\sigma^{\mathrm{T}} \mathbf{n}^{\alpha}$, where the superscript T means matrix transpose. This is equal to $\mathbf{t}^{\alpha}=\boldsymbol{\sigma} \mathbf{n}^{\alpha}$ because of the symmetry $\sigma^{\mathrm{T}}=\sigma$. Subtracting normal stress, the shear traction $\mathbf{s}^{\alpha}$ is obtained:

$$
\mathbf{s}^{\alpha}=\boldsymbol{\sigma} \mathbf{n}^{\alpha}-\left[\left(\mathbf{n}^{\alpha}\right)^{\mathrm{T}} \boldsymbol{\sigma} \mathbf{n}^{\alpha}\right] \mathbf{n}^{\alpha} .
$$

The fault slip to relax the traction acting on the fault plane. Therefore, the slip direction of the $\alpha$ th fault is expected to be in the direction $-\mathbf{s}^{\alpha}$. On the other hand, the actual slip direction is an observable as the slickenlines and sense of the fault. Since the stress $\sigma$ is unknown, the theoretical slip direction $-\mathbf{s}^{\alpha}$ may not coincide with the observed one, so that let $\Delta^{\alpha}$ be the angle between them. The stress tensor can be determined by minimizing the sum of the angular misfits

$$
S=\sum_{\alpha=1}^{N} \Delta^{\alpha} .
$$

The inverse method determines not only the direction of stress axes, but also a parameter that was defined by Lode (1925) is determined:

$$
\mu_{L}=\frac{2 \sigma_{2}-\sigma_{1}-\sigma_{3}}{\sigma_{1}-\sigma_{3}}
$$

where $\sigma_{1}, \sigma_{2}$ and $\sigma_{3}$ are the principal stresses. It is seen that $-1 \leq \mu_{L} \leq 1$, as $\sigma_{1} \geq \sigma_{2} \geq \sigma_{3}$. Lode number $\mu_{L}$ indicates the shape of stress ellipsoid: deviatoric axial compression and tension are indicated by $\mu_{L}=-1$ and +1 , respectively. Intermediate Lode numbers represent triaxial stresses. Note that the present method introduced below is based on Angelier's (1984) who use Bishop's (1966) parameter $\Phi=\left(\sigma_{2}-\sigma_{3}\right) /\left(\sigma_{1}-\sigma_{3}\right)$ rather than $\mu_{L}$. Lode number

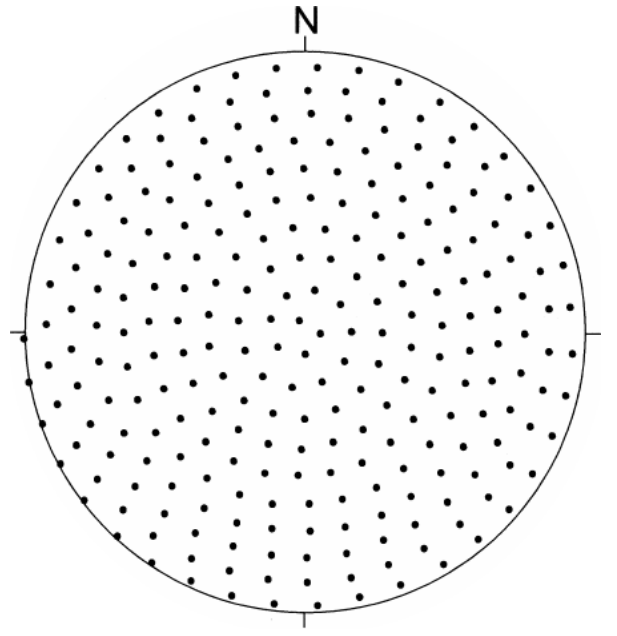

Figure 1: The 256 directions of $\sigma_{3}$ axis used in the inversion. The $\sigma_{1}$ axis is rotated around the $\sigma_{3}$ axis with ca. $11^{\circ}$. Lower-hemisphere, equal-area projection.

is more convenient for this work because both of the axial stresses have the same absolute value $\left|\mu_{L}\right|=1$. The parameters $\mu_{L}$ and $\Phi$ are linearly related: $\mu_{L}=2 \Phi-1$.

Lode number represents the shape of stress ellipsoid, whereas its pose is described by the Euler angles $\theta, \phi$ and $\psi$ of stress axes with respect to geographic frame of reference. Therefore, the inverse calculation is mathematically equivalent with projecting a set of fault-slip data onto a point in a four-dimensional space which $\left(\theta, \phi, \psi, \mu_{L}\right)$ are the coordinates. A point in the parameter space indicates the shape and pose of stress ellipsoid.

\subsection{Multiple inverse method}

The core of the present method is akin to cluster analysis (Menke, 1989), utilizing a sort of self-correlation of the data. Each stress that activated a subset of faults correlate the members of the subset to each other. Suppose that we have $N$ fault-slip data, and that we have no a priori knowledge of how many nor what kind of stress fields are recorded in the data. The problem is how to divide a set of faults into subsets that correspond to stresses that activate the subsets. To solve this problem, we make $k$-element subsets from $N$. It is explained later that $k$ should be larger than or equal to two and $k=4$ or 5 is appropriate for the present method. The number of the subsets composed from the set of $N$ elements is given by the binomial coefficient:

$$
{ }_{N} C_{k}=\frac{N !}{k !(N-k) !}
$$

where $N$ ! stands for the factorial, $N !=N(N-1) \cdots 2 \cdot 1$.

Now, suppose that the faults are classified into two groups that slipped in response to the stress states A and 
$\mathrm{B}$, respectively. The states are represented by two different points, $\mathrm{A}$ and $\mathrm{B}$, in the parameter space. If all faults of a subset belong to the first group, the solution of the inverse calculation applied to the subset should indicate a point at or close to the point $\mathrm{A}$. In case that all the faults belong to group B, the solution would plot near the point B. However, if the subset is a mixture of faults from both groups, the point may be randomly located in the space. Applying the inverse technique to all the subsets, two clusters may appear in the parameter space around the correct answers.

In this study, inverse calculations were performed using a grid search method in the parameter space with the intervals of 2.0/15 for Lode number. The parameter is, accordingly, digitized into $16=2^{4}$ grades from -1 to 1 . Stress axes should be oriented in various directions with uniform angular intervals. However, there is no straightforward way to digitize Euler angles, because the parameter space $(\theta, \phi, \psi)$ is not Euclidean. There is no way to distribute hundreds of points on a sphere with literally equal intervals (Saff and Kuijlaars, 1997), but Rakhamanov et al.'s (1994) algorithm provides a fairly good approximation to the distribution. Accordingly, the algorithm was used to generate the grid points, and set up $256=2^{8}$ directions for the $\sigma_{3}$ axis (Fig. $1)$. As the area of a hemisphere with unit radius is $2 \pi, \sigma_{3}$ axis is varied with an intervals of $(2 \pi / 256)^{1 / 2}$ radians $\approx 9^{\circ}$. The direction of $\sigma_{1}$ axis is rotated around the $\sigma_{3}$ axis with an interval of $180^{\circ} / 2^{4} \approx 11^{\circ}$. Consequently, a total of $65,536=2^{16}$ grid points are used in the inversion.

\section{TEST BY SIMULATED FAULT- SLIP DATA}

The multiple inverse method is applied to artificial faultslip data to demonstrate its validity. Firstly, thirty faults were generated with random orientations (Fig. 2). Secondly, their slip directions were calculated by the Eq. (1) with assumed stress states. Three stresses were assumed: the stress state $\mathrm{A}$ is vertical, axial compression $\left(\mu_{L}=-1\right)$, the state $\mathrm{B}$ is triaxial $\left(\mu_{L}=0\right)$ stress with vertical $\sigma_{1}$ and horizontal $\mathrm{E}-\mathrm{W} \sigma_{3}$ axis. The third case is the heterogeneous fault-slip data- a half of the faults were activated by the stress $\mathrm{A}$ and the remaining 15 faults by the stress B. Thirdly, uniform random noise within $\pm 5^{\circ}$ is added to the pitch angle of slip direction. And, finally, the multiple inverse method was applied to the three cases.

It is necessary to visualize clusters in the fourdimensional parameter space. To do so, the Euler angles $\theta, \phi$ and $\psi$ are converted to the direction of $\sigma_{1}$ and $\sigma_{3}$ axes. The direction of one of the axes is shown by a dot on lower hemisphere, equal-area net and that of the other axis is indicated by a bar attached on the dot: the azimuth and plunge of the other one is indicated by the direction and length of the bar, respectively (Fig. 3). Lode number $\mu_{L}$ is color-

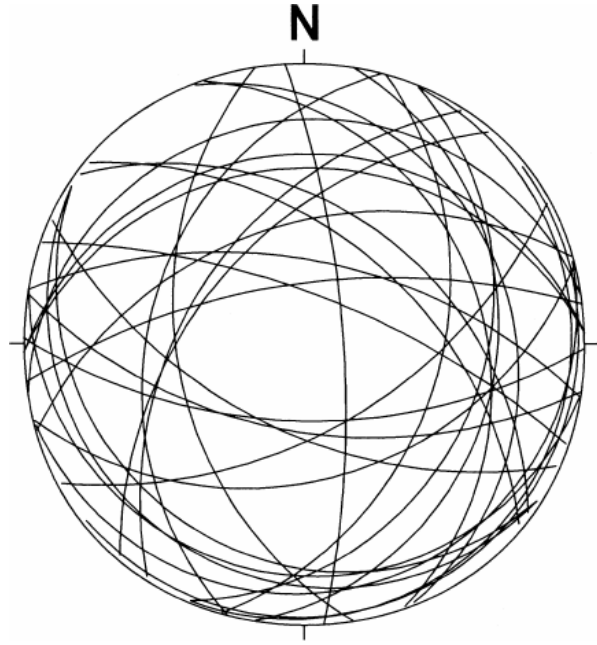

Figure 2: Lower-hemisphere, equal-angle projection showing the direction of fault planes used for simulation.

coded in the plot. By this projection, a solution in the parameter space is shown by a single symbol. Clusters in the four-dimensional space are visualized as a cluster of symbols with the same color, same direction and same length of bars.

The upper panel of Fig. 3 shows the solution for the case 1: all the faults slipped in response to the stress A. The lower panel of the same figure shows those for the case 2: all the faults slipped by the stress B. The correct answers were successfully obtained for the both cases. The purple symbols make a cluster in the upper left panel, indicating axial, vertical compression. In this case, stress state is horizontally isotropic, so that the great-circle girdle of purple dots in the upper right circle of Fig. 3 indicates this stress state. The present method successfully processed the case 3 also, i.e., the heterogeneous fault-slip data generated with the stress state A and B (Fig. 4). Both the stresses are clearly identified. These calculations were performed with $k=4$, and $N=30$, so that the number of solutions is ${ }_{N} C_{k}=27,405$. It is explained later that the number of four is preferential for $k$.

To investigate the density distribution in the parameter space, let $m$ be the number of solutions at a grid point, and $P(m)$ be the frequency that a grid point has $m$ solutions. It is found from the artificial fault-slip data and from filed data, presented below, that the frequency distribution obeys the relationship

$$
P(m)=a m^{-b}
$$

where the parameters $a$ and $b$ depend on data and $k$ (Fig. 5). This is known as Pareto distribution in statistics that applies to, e.g., the population of high income groups - there are a small number of people who have huge incomes (Mood et al., 1974). In this case, this relationship indicates that a 


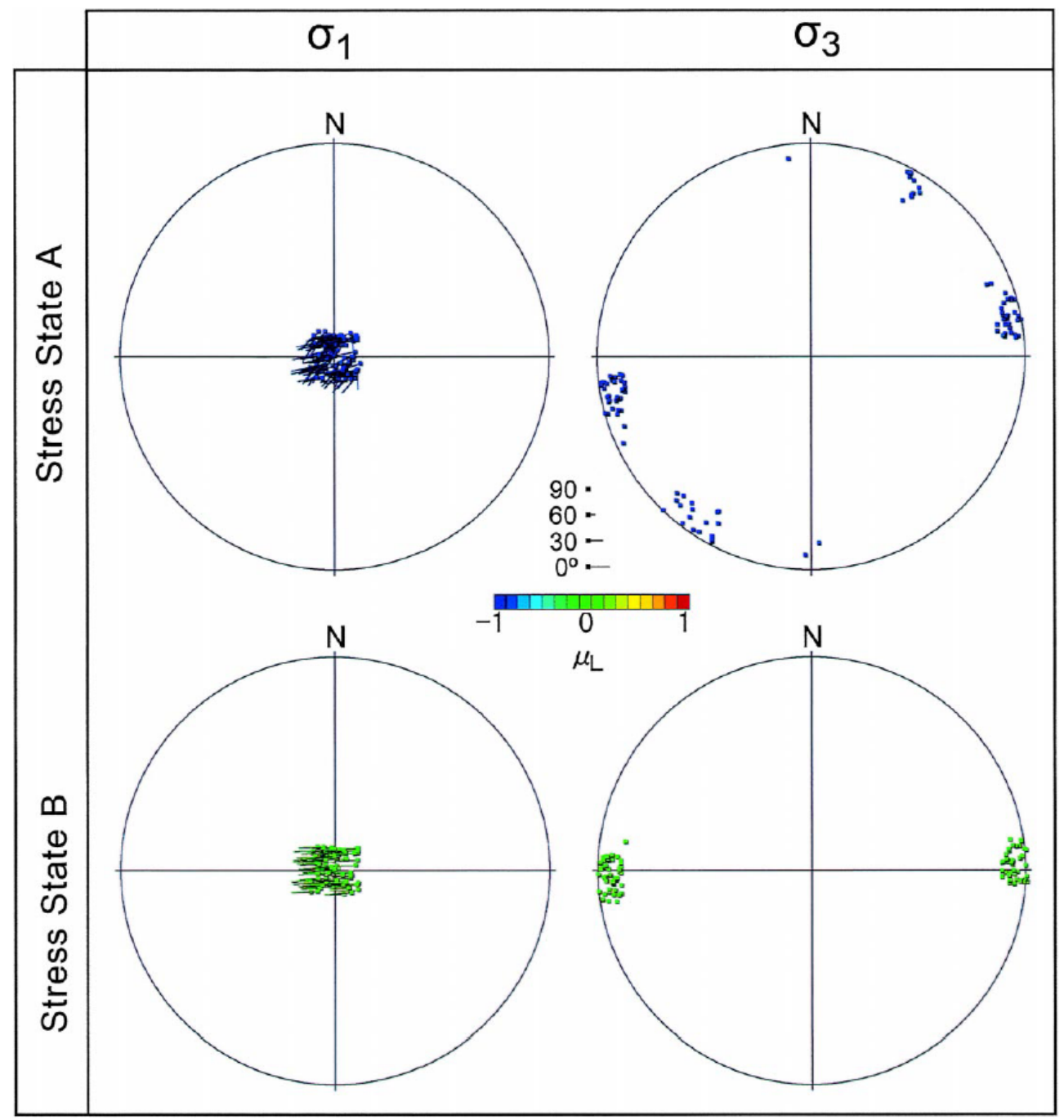

Figure 3: Identified stress states by the multiple inverse method from simulated fault-slip data. A cluster in the fourdimensional space is visualized as a cluster with the same color, same direction and length of bars. In the left panel, the direction of $\sigma_{1}$ axis is shown by a dot on lower hemisphere, equal-area net and that of $\sigma_{3}$ axis is indicated by a bar attached on the dot: the azimuth and plunge of the other one is indicated by the direction and length of the bar, respectively. In the right panel, the projection of the both axes are inverted. The length is proportional to the plunge angle. The Lode number $\mu_{L}$ is color-coded in the plot. By this projection, a solution in the parameter space is shown by a single symbol—a cluster in the four-dimensional parameter space is visualized as a cluster with the same color and the bars with the same direction and length. Multiple inversion was applied with $k=4$, and only solutions with $m \geq 4 s$ is shown, where $s$ is the standard deviation of $m$ 's. The stress A is vertical and axial $\left(\mu_{L}=-1\right)$ compression. The stress B is triaxial $\left(\mu_{L}=0.0\right)$, vertical compression and horizontal, E-W extension. Both the stresses are successfully identified. 
(a) All Solutions

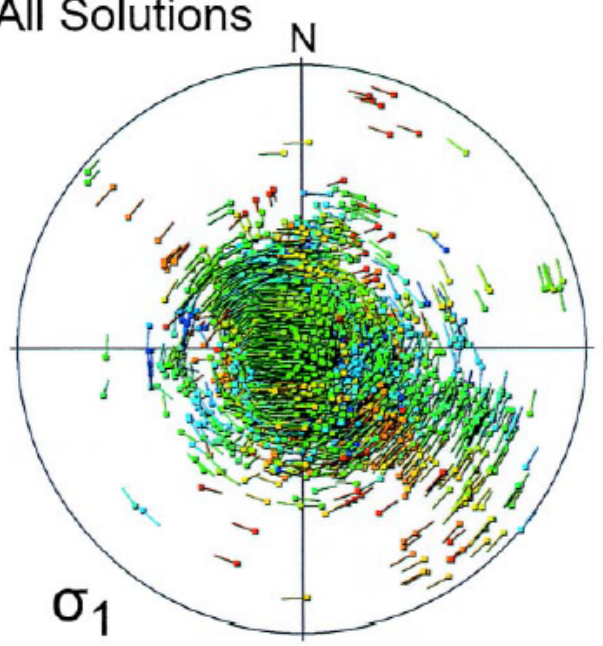

(b) Clusters with $m \geq 6 s$

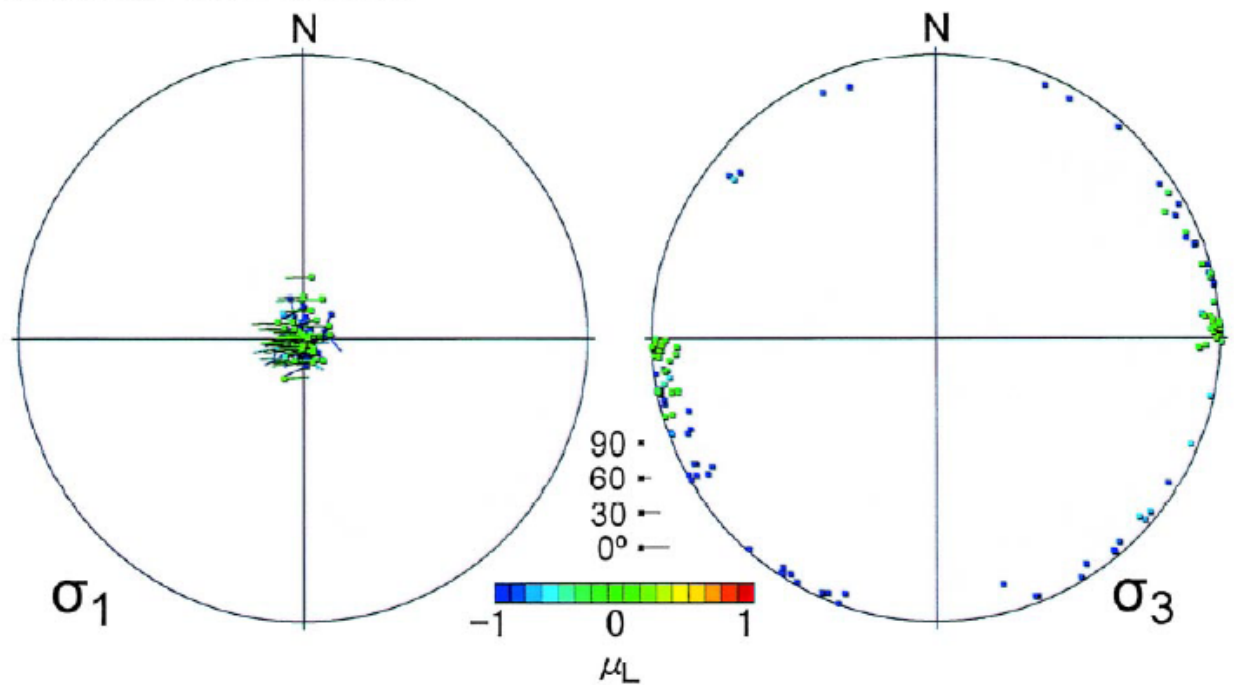

Figure 4: Identified stresses from heterogeneous fault-slip data generated with the stress A and B. The inverse calculations were done with $k=4$. The upper panel shows all the solutions. There are erroneous solutions that are indicated by symbols with warm colors. Those solutions are thinned out in the lower panel by plotting $m / 4 s$ solutions. See text in detail. 
small number of grid points have most of solutions but quite a few points have a few or no solution-clusters appear in the parameter space.

The relationship allows to thin out erroneous solutions and enhance correct ones. Let the italic symbol $s$ be the standard deviation of $P(m)$. Given $m$ solutions at a grid point, a number of $m / 4 s$ symbols were plotted in Fig. 4 (b). If $m / 4 s<1$ at a grid point, no symbol was drawn. The upper panel of Fig. 4 shows all the solutions for the case 3. Erroneous solutions are plotted there, such as warmcolored symbols. However, the correct solutions appear as densest clusters. In the lower panel of Fig. 4, only $m / 4 s$ solutions are plotted. The result is that the correct solutions are enhanced, though a small number of erroneous ones still remain.

\subsection{Convergence of solutions}

The choice of the parameter $k$ is arbitrary, yet smaller $k$ 's may yield erroneous and unstable solutions. As data always include noise, the inverse calculation for a small number of faults sometimes provides improper answers. We refer this as the stability of solution. On the other hand the amount of computation inflates with the increase of $k$ in the speed ${ }_{N} C_{k} \propto N^{k}$, if $k \ll N$. A small $k$ is preferable for the reduction of execution time of computation. How much is the appropriate value for $k$ ?

Varying $k$ from 2 to 7 , the present method was applied to the simulated heterogeneous fault-slip data. If $k=2$, correct solutions do not make clusters at all. However, they do appear, if $k \geq 3$ (Fig. 6). The solutions for $k=4$ and 5 are nearly identical; the gross pattern in the parameter space shows convergence. However, those for $k=3$ is slightly different: erroneous solutions remain, such as the green dots along the base circle. In this case, consequently, $k=4$ is the optimal choice.

\section{APPLICATION TO FIELD DATA}

\subsection{Data}

The multiple inverse method is applied to 25 reverse faults that dislocate the Tano Formation, Miyazaki Group, western Japan (Fig. 7a). The formation is correlated to the uppermost Miocene (Suzuki, 1987). All the faults have displacements less than a few centimeters and diagonally cut a 20-cm-thick sandstone layer.

The faults were selected, because the following observations suggest that they record relatively simple stress fields. (1) Reverse faults are rare in the area, although most of faults in the Group have normal or oblique normal sense of slip (Tokushige and Fabbri, 1996). (2) Only the 25 faults have calcitic slickenfiber lineations among hundreds of meso-scale fault observed in the Miyazaki area. The

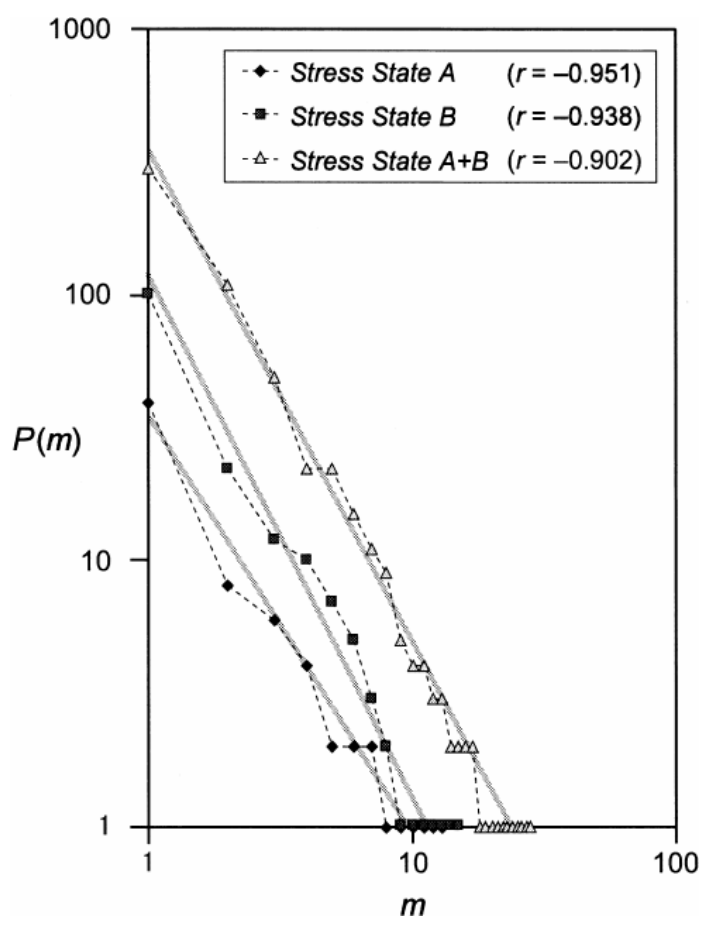

Figure 5: The log-log plot for the frequency distribution $P(m)$ for the simulated fault-slip data. Gray lines show regression lines. Correlation coefficients, $r$, are indicated also. 
(a) $k=2$

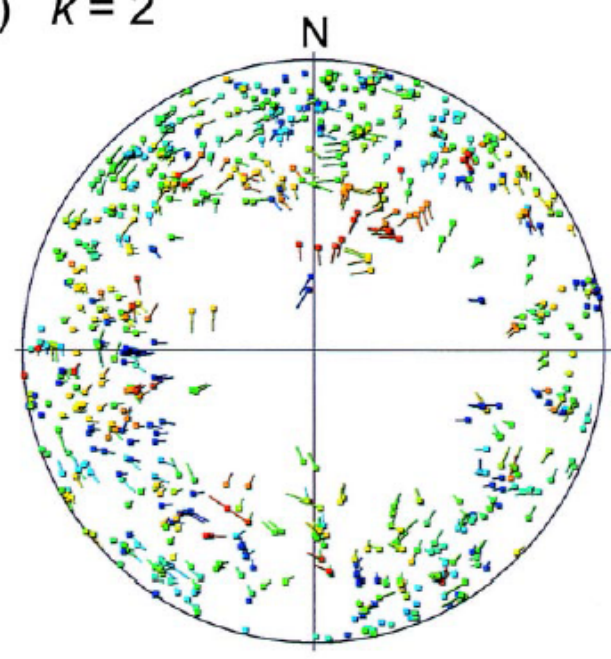

(c) $k=4$

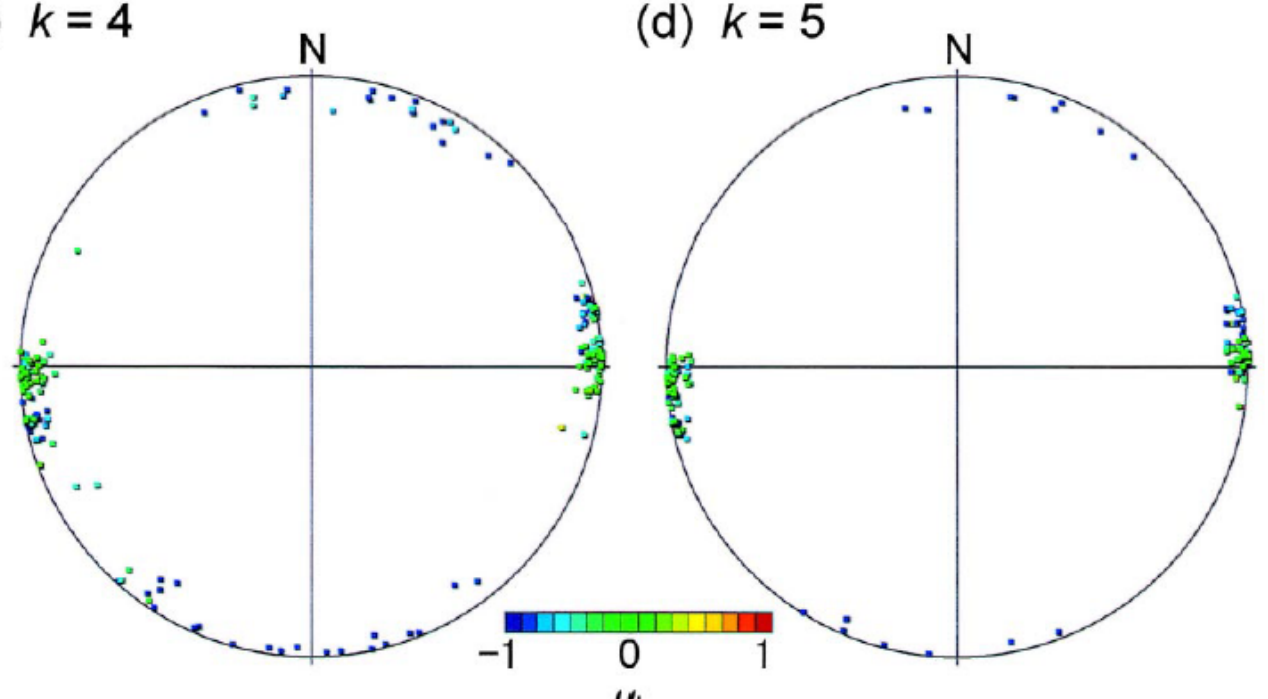

(b) $k=3$

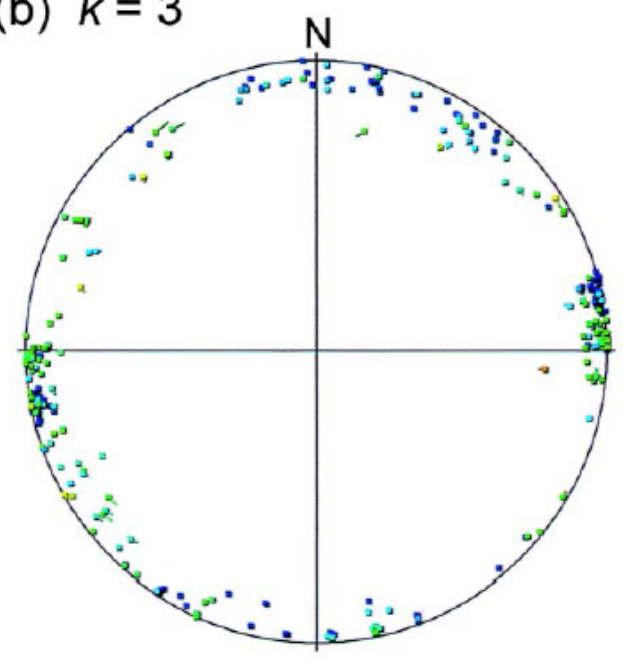

(d) $k=5$

Figure 6: Comparison with solutions calculated with various $k$. The direction of $\sigma_{3}$ axes calculated from the heterogeneous fault-slip data are shown by dots. Lower hemisphere, equal area projection. The length and direction of bar attached on the dot show the plunge and azimuth of $\sigma_{1}$ axis. The multiple inversion with $k=2$ yields no correct answer, whereas correct ones are obtained with $k \geq 3$. Solutions with $k=3$ includes erroneous one such as orange dot in the SW quadrant. A number of $m / 4 s$ symbols are plotted. 

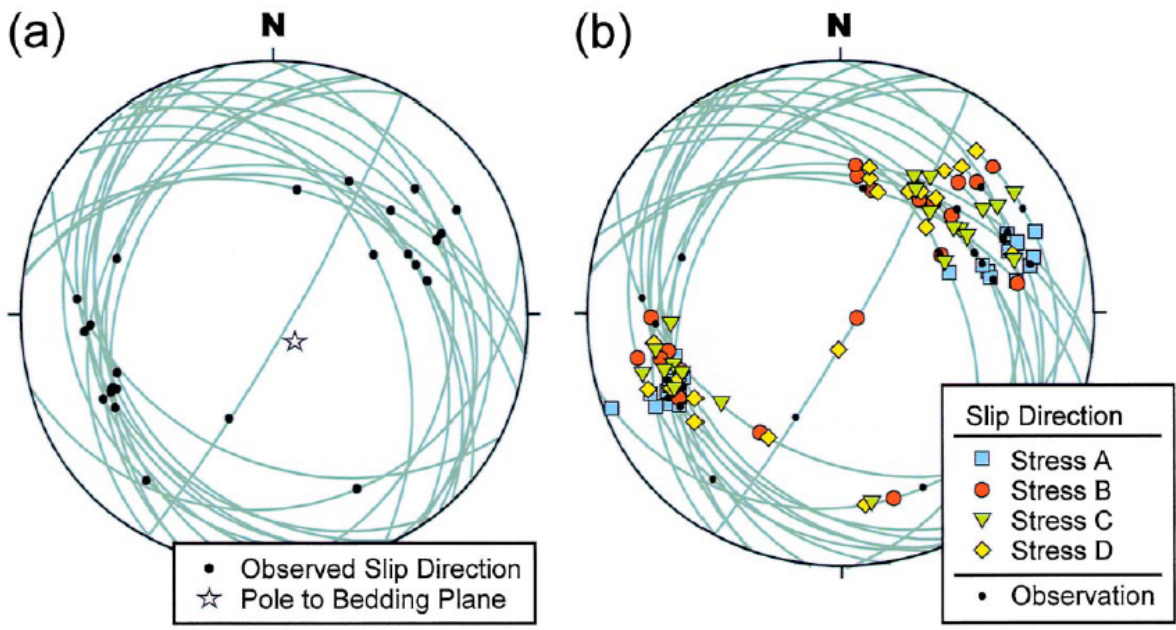

Figure 7: (a) Reverse and oblique-reverse faults observed at an outcrop of the Tano Formation, Miyazaki Group. Lower hemisphere, equal angle projection. Solid circles indicates the slip direction of foot walls. (b) Theoretical slip directions by the stress A trough D that were identifed by the multiple inverse method from the fault-slip data shown in (a).

fibers indicate that their growth was coeval with slow fault movement (Twiss and Moores, 1992). Therefore, this and the first observations narrows the period(s) of activity. (3) The object data were collected at an outcrop 10 meters in width (lat. $31^{\circ} 50^{\prime} 20^{\prime \prime} \mathrm{N}$, lon. $131^{\circ} 23^{\prime} 10^{\prime \prime} \mathrm{E}$ ), so that they represent stress(es) at one locality.

Most of the faults are dip-slip faults with slip direction in the NE and SW quadrants (Fig. 7a), suggesting that they are a conjugate set. Extensional stress in ENE-WSW direction is the most probable solution. Of course, there is an apparent exception - the high angle, sinistral fault. In addition, the trend of the faults has a large divergence, suggesting that conjugate faulting alone cannot account for the whole data.

\subsection{Results}

The multiple inverse method is applied with $k$ from 2 to 7 in order to see convergence (Fig. 8). If they are conjugate set, the $\sigma_{1}$ axis must lie in a NE-SW direction. The solution with $k=2$ already show such an stress state, but has a lot of $\sigma_{1}$ axes distributed along the base circle. The increase of $k$ makes clusters more coherent. The gross pattern becomes stationary for $k \geq 5$. The convergence indicates that $k=5$ is the optimal choice for the parameter $k$.

The stable and coherent clusters with $k=7$ are labeled in Fig. 9. The cluster $\mathrm{C}$ of green symbols corresponds to the conjugate faulting. The color indicates an intermediate Lode number at $\mu_{L} \approx 0.0$. Accordingly the cluster indicate a triaxial stress with ENE-WSW trending $\sigma_{1}$ and vertical $\sigma_{3}$ axes. However, the trend of the faults has a large divergence, resulting in the appearance of other clusters. The cluster D is composed of yellow symbols, indicating a mod- erately high Lode number. Not only these triaxial stresses, but axial stresses A and B are identified. They are represented by blue and reddish symbols, respectively. The stress A is ENE-WSW trending, axial deviatoric compression. The stress B is vertical, axial, deviatoric tension.

\subsection{Interpretation}

Four stresses were identified from the Miyazaki data. They are equally plausible solutions. Fig. 7 (b) shows the theoretical slip directions of the faults by the stresses. The directions are roughly parallel to the observed ones, however, those by the stress D have larger misfits than others. Therefore, the stress D is the least significant solution.

The stresses A, B and C are equally significant. All inverse methods, including the present one, cannot distinguish which stress was real. The fact is that the three solutions are natural consequences when we process conjugate faults by the inverse method that minimize the sum of angular misfits. In order to show this, let us define a Cartesian coordinate whose $x, y$, and $z$ axes are oriented in north, east, and downward, respectively (Fig. 10). In addition, let $\delta$ be the identity tensor. Even if the stress tensor $\sigma$ is replaced by $p \sigma+q \delta$ where $p$ and $q$ are arbitrary, the slip direction predicted by Eq. (1) is not affected. Therefore, the results of the inverse method has uncertainty corresponding to $p$ and $q$. Accordingly, we assume three stress tensors:

$$
\begin{gathered}
\sigma^{(1)}=\left(\begin{array}{ccc}
1 & 0 & 0 \\
0 & 0 & 0 \\
0 & 0 & -1
\end{array}\right), \quad \sigma^{(2)}=\left(\begin{array}{ccc}
1 & 0 & 0 \\
0 & 0 & 0 \\
0 & 0 & 0
\end{array}\right), \\
\sigma^{(2)}=\left(\begin{array}{lll}
1 & 0 & 0 \\
0 & 1 & 0 \\
0 & 0 & 0
\end{array}\right) .
\end{gathered}
$$




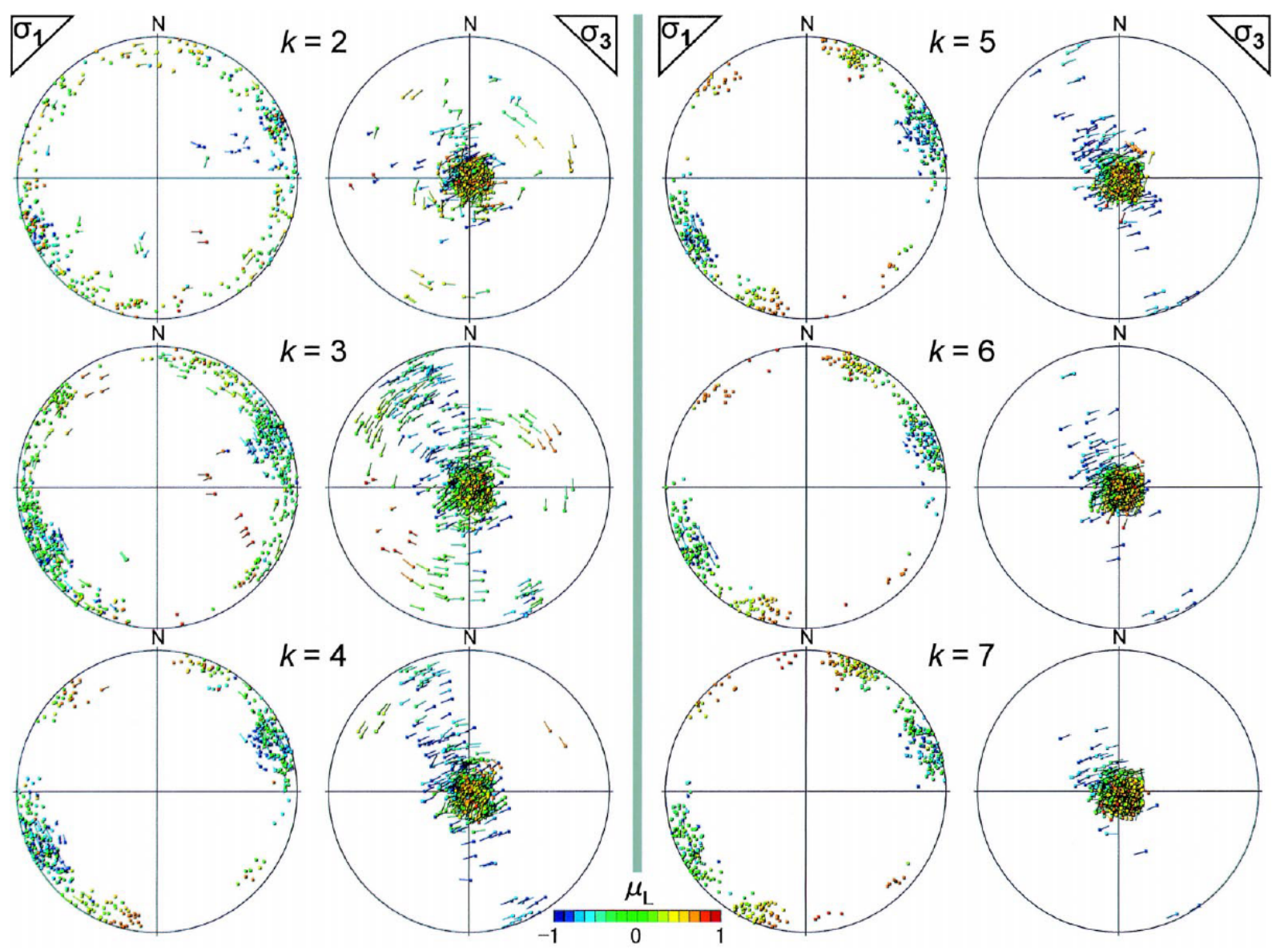

Figure 8: Convergence of clusters from $k=2$ to 7 for the Miyazaki data (Fig. 7). The gross pattern does not change for $k \geq 4$.

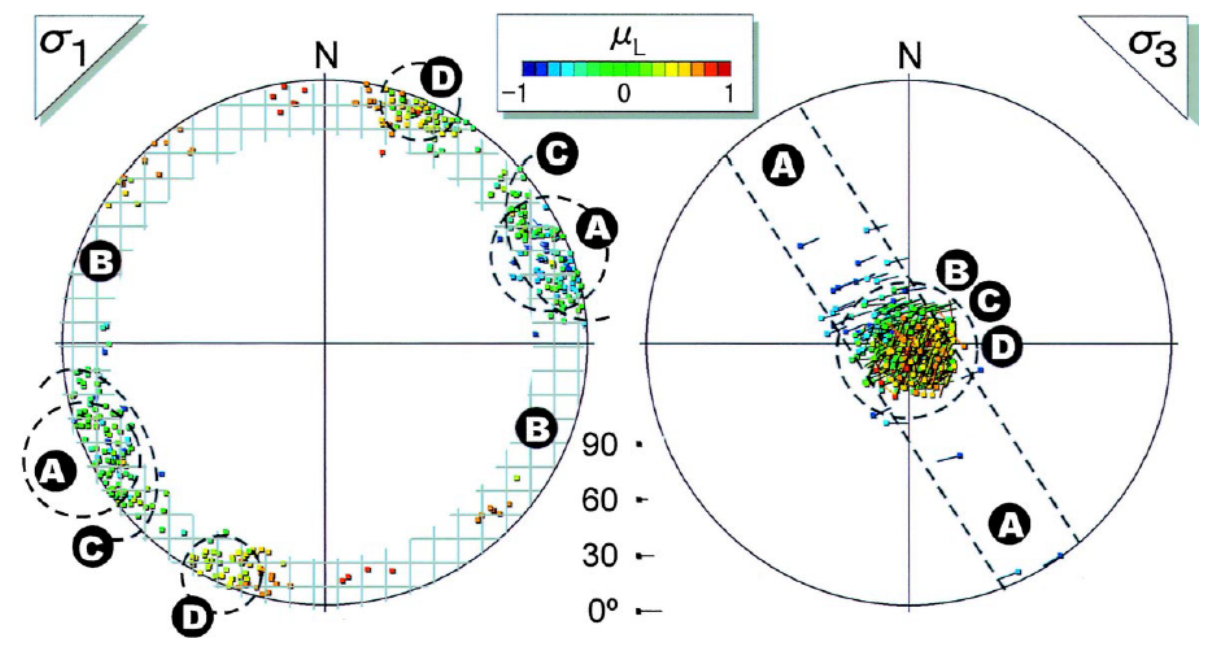

Figure 9: Clusters obtained by the multiple inverse method applied to the Miyazaki data with $k=7$ and $e=6$. The direction of $\sigma_{1}$ and $\sigma_{3}$ axes is shown by left and right lower-hemisphere Schumidt net, respectively. Dot-and-bar symbols: see caption in Fig. 3. The stresses A, B, and C are equally plausible solutions. The cluster of reddish symbols in the cross-hatched area labeled as B indicates a vertical, axial, deviatoric tensional stress. 
The tensor $\sigma^{(1)}$ represents a stress with $\mu_{L}=0$ and N-S compression and vertical extension. Anderson theory of faulting predicts that this stress can give rise to $\mathrm{E}-\mathrm{W}$ tending conjugate faults that dip at about $30^{\circ}$. The tensors $\sigma^{(2)}$ and $\sigma^{(3)}$ represent axial stresses. The former is a horizontal, $\mathrm{N}-$ $\mathrm{S}$ trending, axial compression $\left(\mu_{L}=-1\right)$. The latter, $\sigma^{(3)}$, represents a vertical, axial, and deviatoric tension $\left(\mu_{L}=1\right)$.

Suppose a preexisting fracture which is subparallel to one of the expected conjugate faults. The attitude of the fracture is denoted by its azimuth of dip direction (Fig. 10). If the fracture is activated by one of the stresses, the slip direction is readily calculated by Eq. (1) such that

$$
\mathbf{s}^{(i)}=\boldsymbol{\sigma}^{(i)} \mathbf{n}-\left[\mathbf{n}^{\mathrm{T}} \boldsymbol{\sigma}^{(i)} \mathbf{n}\right] \mathbf{n}
$$

where $i=1,2$ or 3 . The vector $\mathbf{s}^{(i)}$ stands for the slip direction of the fracture due to the stress $\sigma^{(i)}$.

Fig. 10 shows the slip directions with various fracture azimuth with fixed stress axes. The triaxial stress $\sigma^{(1)}$ causes slip in the $\mathbf{s}^{(1)}$ direction in the figure. This direction is nearly parallel to the direction $\mathbf{s}^{(2)}$ that indicates the slip direction due to the axial compression $\sigma^{(2)}$. If the fault has an E$\mathrm{W}$ trend, the other axial stress $\sigma^{(3)}$ can move the fault in the similar direction. Therefore, the three stresses activate the preexisting fractures in a similar manner if the fracture planes are nearly parallel to the conjugate faults. It further means that stress inversion cannot determine which stress is valid. The inversion provide three solutions from conjugate faults.

Stress inversion, not only the multiple inverse method but also the classic inverse method of Angelier (1984), can determine possible stresses consistent with observed fault-slip data. As the multiple inverse method has more resolution than the classic method especially in processing heterogeneous data, the plural solutions are differentiated. A fault collection that seems a conjugate set results in such three stresses. We call them associated solutions determined from conjugate faults. The cluster $\mathrm{A}$ and $\mathrm{C}$ have $\sigma_{1}$ axes in the same direction, so that they are associated to each other. By contrast, the cluster of yellow symbols has a higher Lode number than the green one, so that the stress indicated by the yellow cluster is independent from the other solutions. As a result, the Miyazaki data require at least two stresses $\mathrm{C}$ and $\mathrm{D}$ to account for the fault movements. One, two or all of the stress A, B, and C affected the faults.

It is important that the validity of associated stresses is determined by field observations other than the fault-slip data used in the inverse method or by some theoretical models such as minimum dissipation principle.

When the simulated fault-slip data were processed in the previous section, the correct solutions were determined successfully. The processing was successful because the faults have various orientations. By contrast, the faults observed in Miyazaki have similar orientations. These observations

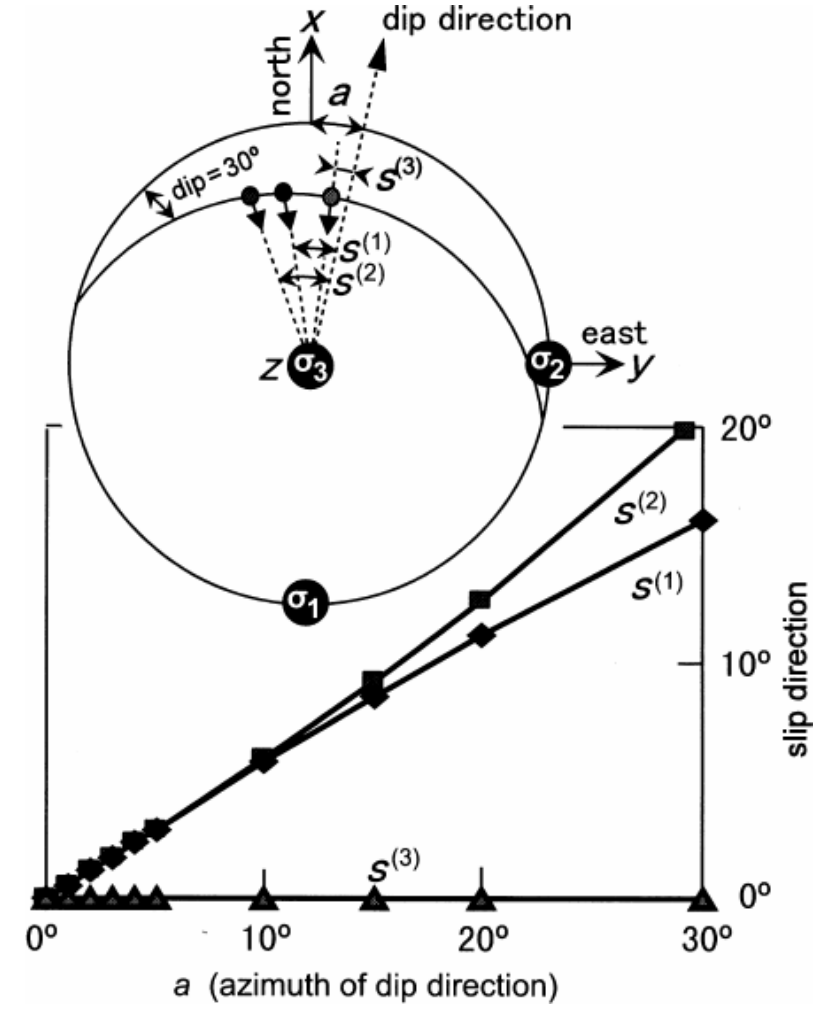

Figure 10: Slip directions $\mathbf{s}^{(1)}, \mathbf{s}^{(2)}, \mathbf{s}^{(3)}$ predicted from Eq. (1) by the stresses $\sigma^{(1)}, \sigma^{(2)}$, and $\sigma^{(3)}$, respectively. The direction of the fault plane is indicated by the azimuth of dip direction, $a$, with the dip fixed at $30^{\circ}$. Note that the stresses cause an identical slip direction if $a=0^{\circ}$.

teach us that a narrow range of fault orientations results in a poor resolution.

\section{DISCUSSION}

Several researchers have presented inverse methods to separate stresses from heterogeneous fault-slip data. Tokushige and Fabbri (1996) attempted to identify paleo stresses from heterogeneous fault-slip data that were collected from the Miyazaki district. They used the right dihedra method (Angelier and Mechler, 1977). The method is applied to the Miyazaki data shown in Fig. 7. The result is shown in Fig. 11 (a). The probability distribution of stress axes determined by the right dihedra method indicates only one stress state with vertical $\sigma_{3}$ and horizontal $\sigma_{1}$ axes in the NE and SW quadrants.

Armijo et al. (1982) apply inverse calculation recursively to those subset of data that show large misfit from slip directions predicted from formerly determined stresses. The first inversion is applied to the whole fault collection. Mino and Yamaji (1999) applies this approach to Quater- 
(a)

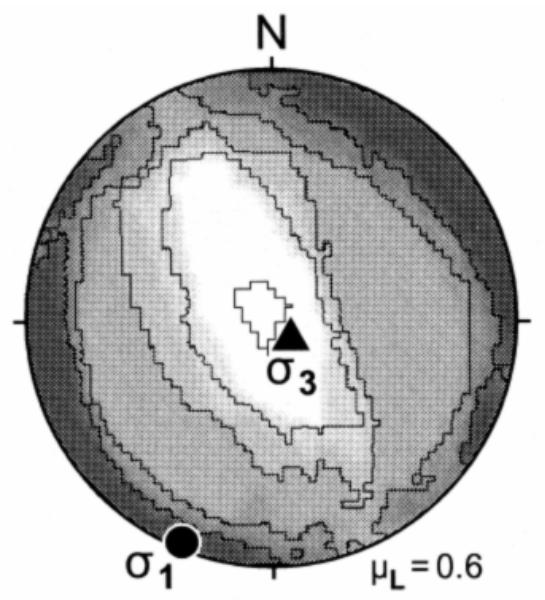

(b)

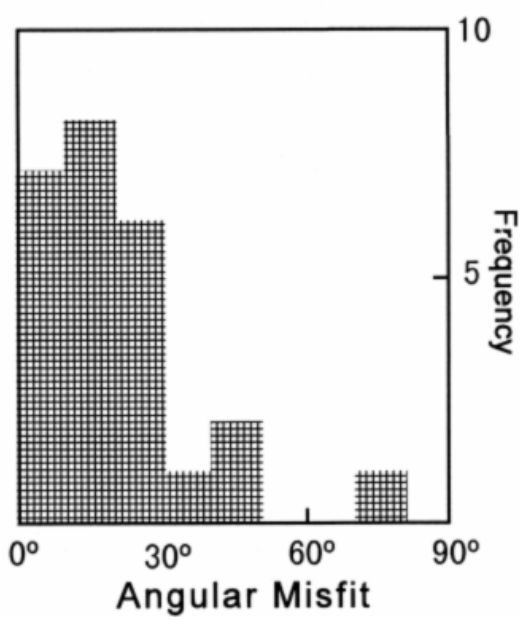

Figure 11: (a) The fault-slip data shown in Fig. 7 is processed by the right dihedra and Angelier's (1979) classic inverse methods. The result is a NNE-SSW trending, triaxial $\left(\mu_{L}=0.6\right)$ tension, which is identical with the stress D obtained by the multiple inverse method. (b) The histogram of the angular misfit the observed slip direction with that predicted by Eq. (1) with the stress, showing a unimodal distribution with a bload tail. If the histogram had a minimum, the fault-slip data could be divided into two groups and other stresses could be determined by the classic method. The data is formidably heterogeneous for the classic inverse method.

nary mesoscale faults in central Japan. Their first inversion by classic Angelier's (1979) method resulted in bimodal distribution of angular misfits, allowing Mino and Yamaji to divide the data set into two groups.

The same approach was taken to process the Miyazaki data (Fig. 7) to compare the results of the multiple inverse method. However, the Angelier's (1979) method resulted in the single-mode frequency distribution with a broad tail toward large misfits (Fig. 11), suggesting that the optimal stress alone does not account for the whole fault data. However, the distribution make the division of data set difficult. The obtained stress has a moderately high Lode number $\left(\mu_{L}=0.6\right)$ and with NNE-SSW trending $\sigma_{1}$, and vertical $\sigma_{3}$ axes. This is concordant with the stress D that was obtained by the multiple inverse method. Therefore, the recursive method did not work well for the Miyazaki data.

In principle, the recursive method has several difficulties. (1) Given heterogeneous data, we cannot expect to obtain the correct solution for the first inversion. The sum of angular misfits, $S$, or any other measure of misfits, has a lot of local minima in the parameter space. The minimum point is not always determined by the most significant stress. Therefore, it is not easy to obtain the correct solution at the first inversion. (2) It is sometimes difficult to find a trough in the histogram of misfits to divide data for the following iteration. In addition, (3) data are subdivided many times and later inversions use small parts of them, resulting in unstable solutions. The reliability of detected stresses depend on, therefore, the order they detected.

By contrast, solutions are treated equally in the present method. It is fully automatic and does not require uses' choice or decision during data processing. The present method deals with stresses equally. The orthodox recursive method does not. The inequality makes the latter technique unreliable when the second or later solution is calculated. Moreover, it is sometimes difficult for the recursive method to obtain correct solution at the first iteration.

Acknowledgments - The present method stemmed from the discussions with S. R Wallis. Special thanks go to O. Fujiwara, T. Fukuma, I. Hagiwara, and H. Kuroki for the assistance to collect field data. I thank A. Okada, F. Masuda, and K. Takemura for discussion and encouragement. Thanks are also due to J. M. Fletcher, L. Arlegui, A. F. NietoSamaniego and T. Shimamoto for critical comments and the improvement of the manuscript. Funded by the Grant-inAid for Scientific Research (08640568 and 08304033) from the Ministry of Education, Science and Culture, Japan.

\section{REFERENCES}

Angelier, J., 1979. Determination of the mean principal direction of stresses for a given fault population. Tectonophysics 56, T17-T26.

Angelier, J., 1984. Tectonic analysis of fault slip data sets. Journal of Geophysical Research 89, 5835-5848 .

Angelier, J. and Huchon, P., 1987. Tectonic record of con- 
vergence changes in a collision area: the Boso and Miura peninsulas, central Japan. Earth and Planetary Sciences Letters 81, 397-408.

Angelier, J. and Mechler, P., 1977. Sur une méthode graphique de recherche contraintes principales également utilisable en tectonique et en séismologie: la méthode des dièdres droits. Bulletin de la Societe Géologique de France 19, 1309-1318.

Armijo, R., Carey, E. and Cisternas, A., 1982. The inverse problem in microtectonics and the separation of tectonic phases. Tectonophysics 82, 145-160.

Bishop, A. W., 1966. The strength of solids as engineering materials. Geotechnique 16, 91-130.

Carey, M. E. and Brunier, M. B., 1974. Analyse theorique et numerique d'um modele mecanique elementaire applique a l'etude d'une populationde failles. Comptes Rendus Hebdomadaires des Seances de l'Academie des Sciences 279(D), 891-894.

Cashman, P. H. and Ellis, M. A., 1994. Fault interaction may generate multiple slip vectors on a single fault surface. Geology 22, 1123-1126.

Engelder, T., 1993. Stress regimes in the lithosphere. Princeton Univ. Press, Princeton.

Etchecopar, A., Vasseur, G. and Daignieres, M., 1981. An inverse problem in microtectonics for the determination of stress tensors from fault striation analysis. Journal of Structural Geology 3, 51-65.

Fry, N., 1999. Striated faults: visual appriciation of their constraint on possible paleostress tensors. Journal of Structural Geology 21, 7-21.

Gephart, J. W. and Forsyth, D. W., 1984. An improved method for determining the regional stress tensor using earthquake focal mechanism data: Application to the San Fernando Earthquake sequence. Journal of Geophysical Research 89, 9305-9320.

Guatteri, M. and Spudich, P., 1998. coseismic temporal changes of slip direction: the effect of absolute stress on dynamic rupture. Bulletin of Seismological Society of America 88, 777-789.

Huang, Q., 1988. Computer-based method to separate heterogeneous sets of fault-slip data into subsets. Journal of Structural Geology 10, 278-299.

Lode, W., 1925. Versuche über den Einfluß der mittleren Hauptspannung auf die Fließgrenze. Zeitschrift für angewandte Mathematik und Mechanik 5, 142-144.
Marrett, R. and Allmendinger, R. W., 1990. Kinematic analysis of fault-slip data. Journal of Structural Geology 12, 973-986.

Menke, W., 1989. Geophysical data analysis: discrete inverse theory. Academic Press, San Diego.

Mino, K. and Yamaji, A., 1999. The separation of paleostresses from heterogeneous fault-slip data: the case of Boso area, Japan. Journal of Geological Society of Japan 105, 574-584.

Mood, A. M., Graybill, F. A. and Boes, D. C., 1974. Introduction to the theory of statistics, 3rd ed. McGrawHill, New York.

Nieto-Samaniego, A. F. and Alaniz-Alvarez, S. A., 1997. Origin and tectonic interpretation of multiple fault patterns. Tectonophysics 270, 197-206.

Otsuki, K., Minagawa, J., Aono, M. and Ohtake, M., 1997. On the curved striations of Nojima seismic fault engraved at the 1995 Hyogoken-Nambu earthquake, Japan. Journal of the Seismological Society of Japan 49, 451-460.

Pollard, D. D., Saltzer, S. D. and Rubin, A. M., 1993. Stress inversion methods; are they based on faulty assumptions? Journal of Structural Geology 15, 10451054.

Rakhamanov, D. E. A., Saff, E. B. and Zhou, Y. M., 1994. Minimal discrete energy on the sphere. Mathematical Research Letters 1, 647-662.

Reches, Z., 1978. Analysis of faulting in threedimensional strain field. Tectonophysics 47, 109-129.

Saff, E. B. and Kuijlaars, A, B. J., 1997. Distributing many points on a sphere. Mathematical Intelligence 11, 511.

Suzuki, H., 1987. Stratigraphy of the Miyazaki Group in the southeastern part of Miyazaki Prefecture, Kyushu, Japan. Contribution of the Institute of Geology and Paleontology, Tohoku University, 90, 1-24.

Tokushige, H. and Fabbri, O., 1996. Mesofaults and associated stress field in the Late Miocene to Pliocene forearc deposits of the Miyazaki district, southeast Kyushu, Japan. Journal of Geological Society of Japan 102, 622-634.

Twiss, R. J. and Moores, E. M., 1992. Structural geology. W. H. Freeman and Company. New York.

Twiss, R. J. and Unruh, J. R., 1998. Analysis of fault slip inversions; do they constrain stress or strain rate? Journal of Geophysical Research 103, 12205-12222. 
Yoshida, S., Koketsu, K., Shibazaki, B., Sagiya, T., Kato, T. and Yoshida, Y., 1996. Joint inversion of nearand far-field waveforms and geodetic data for the rupture process of the 1995 Kobe earthquake. Journal of Physics of the Earth 44, 437-454. 